\title{
Abcès phœnix : démarche therapeutique au centre de consultations et de traitements odonto-stomatologiques (CCTOS) d'Abidjan
}

\section{Phœnix abscess: treatment modalities in odonto-stomatology service (CCTOS) of Abidjan}

AKPE JONAS ADOU ${ }^{1}$, PATRICE KOUAME², MARIE CHANTAL AVOAKA ${ }^{2}$, FLORENT KOUAKOU ${ }^{1}$, EDMEE ABOUATTIER-MANSILLA ${ }^{1}$

\section{RÉSUMÉ}

Un abcès phœnix provoqué par la nécrose septique de la pulpe d'une incisive centrale maxillaire a été traité au CCTOS d'Abidjan. La démarche thérapeutique a combiné un traitement endodontique non chirurgical à base d'hydroxyde de calcium $\left[\mathrm{Ca}\left(\mathrm{OH}_{2}\right)\right.$ + sérum physiologique] et une médication générale à base d'antibiotique. L'usage de l'hydroxyde de calcium après un drainage transcanalaire de l'abcès a permis la guérison de la lésion. Med Buccale Chir Buccale 2007; 13 : 161-165.

mots clés: abcès phœnix, endodontie, hydroxyde de calcium, antibiotique

\section{SUMMARY}

médecine

buccale chirurgie buccale

VOL. $13, N^{\circ} 3$ 2007

page 161

A case and treatment in odontostomatology service (Abidjan) of phoenix abscess caused by necrotic pulp of maxillary central incisor is being presented. Nonsurgical endodontic therapy, complimented by antibiotic, is the treatment modalities of this case. For the treatment calcium hydroxide powder mixed with physiological serum was used. Usage of calcium hydroxide paste after pus was removed through standard endodontic access cavity was leaded to successful healing of phoenix abscess. Med Buccale Chir Buccale 2007; 13 : 161-165.

key words: phœnix abscess, endodontic treatment, calcium hydroxide, antibiotic 
L'abcès phœnix ou abcès récurrent traduit l'exacerbation aiguë d'une pathologie inflammatoire chronique d'origine endodontique qui se développe à partir du parodonte profond périradiculaire. La poussée infectieuse ou " flare up » présente le même tableau clinique que la parodontite apicale aiguë abcédée [14]. Le diagnostic différentiel repose sur l'examen radiographique qui montre dans le cas d'un abcès phœnix, une image radioclaire périradiculaire. Différents facteurs peuvent expliquer la réactivation infectieuse: - passage forcé de débris nécrotiques, de bactéries et/ou d'endotoxines au-delà du foramen apical au cours d'une manœuvre instrumentale, - diminution des résistances du patient : déficit immunitaire, diabète, fatigue, stress [14].

Cette lésion est caractérisée par l'accumulation de pus dans la région du périapex et une douleur violente.

médecine

buccale

chirurgie

buccale

VOL. $13, N^{\circ} 3$ 2007

page 162

En Côte d'Ivoire, les consultations dentaires sont tardives et ont, le plus souvent, pour motif la douleur. La prise en charge des lésions inflammatoires parodontales abcédées se résume à un traitement radical : suppression de l'organe responsable de la souffrance, c'est-à-dire la dent causale [2]. Or l'évolution des techniques et des matériaux en endodontie font qu'il est, aujourd'hui possible pour un plus grand nombre de praticiens, d'éviter les avulsions dentaires.

Ce travail propose aux praticiens, une alternative à l'extraction de la dent atteinte d'un abcès phœnix : le traitement endodontique associé à une antibiothérapie.

\section{CAS CLINIQUE}

\section{Examen clinique et radiographique et traitement}

Madame K. S. A. âgée de 22 ans se présente à la consultation du CCTOS. Elle se plaint d'une douleur spontanée, sourde, intense, continue, localisée dans la région des incisives supérieures, d'une sensation de dent trop longue et elle signale une tuméfaction récurrente en regard de la 21. L'état général est altéré (fièvre, adénopathie, asthénie). Les tests de vitalité pulpaire sont négatifs. La percussion axiale et la palpation apicale de la 21 sont très douloureuses. La dent présente une mobilité de type II et

une importante cavité de carie qui communique avec la chambre pulpaire. L'examen radiographique retroalvéolaire montre une image radioclaire périapicale sur la 21 (Fig. 1). Ce tableau est caractéristique d'un abcès récurrent (abcès phœnix) sous-périosté d'origine endodontique [10].

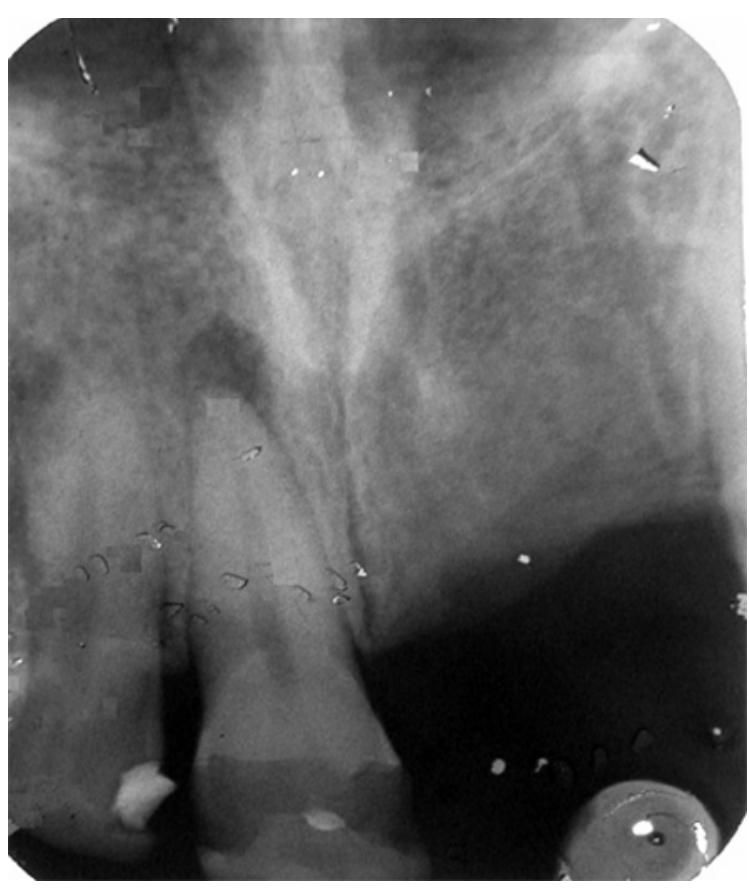

Figure 1 : Radiographie préopératoire ; lésion apicale. Diagnostic X-ray ; periapical lesion.

Comme il n'y avait aucune contre-indication à un traitement endodontique, la démarche thérapeutique a donc combiné un traitement endodontique à base d'hydroxyde de calcium et une antibiothérapie systémique. Au cours de la première séance, le protocole d'urgence a été appliqué [17]: le site d'intervention désinfecté au Dakin, la chambre pulpaire de la dent ouverte, la digue mise en place puis on a effectué un cathétérisme et un drainage transcanalaire partiel (préparation manuelle jusqu'à la lime $25 / 100$, irrigation à l'hypochlorite de sodium à 2,5\%). Un meulage coronaire sélectif a été réalisé, la dent laissée ouverte et l'association amoxicilline et acide clavulanique (2g par jour pendant 7 jours) et des bains de bouche à base de chlorhexidine (3 fois par jour) ont été prescrits Les séances suivantes ont été ainsi programmées :

- $1^{\text {re }}$ séance $(\mathrm{J}+0)$ : pose de la digue, détermination de la longueur de travail, préparation séquentielle manuelle jusqu'à la lime 30/100, irrigation à 
l'hypochlorite de sodium 2,5\%, séchage du canal à l'aide de pointes de papier absorbant, médication canalaire temporaire extemporanée ; poudre d'hydroxyde de calcium + sérum physiologique (consistance crème, mise en place à l'aide d'un bourre-pâte de Lentulo), obturation coronaire provisoire au ciment de verre ionomère.

- $2^{e}$ séance $(\mathrm{J}+2)$ : le drainage est obtenu et une amélioration des signes locorégionaux et généraux est observée. Le protocole opératoire d'apexification (tentative d'occlusion foraminale) est appliqué. - $3^{e}$ séance $(\mathrm{J}+8)$ : pose de la digue, parage, irrigation, séchage, hydroxyde de calcium, obturation coronaire provisoire au ciment de verre ionomère.

- $4^{e}$ séance $(J+15)$ : disparition des signes cliniques, pose de la digue, parage, irrigation, séchage, obtention d'un canal propre, sec et d'une matrice apicale. L'obturation du canal est réalisée par une technique fiable ; la condensation latérale à froid de gutta percha. Sa densité permet d'assurer la prolongation de l'antisepsie canalaire. Une restauration coronaire étanche au composite est mise en place pour éviter toute nouvelle contamination de l'endodonte.

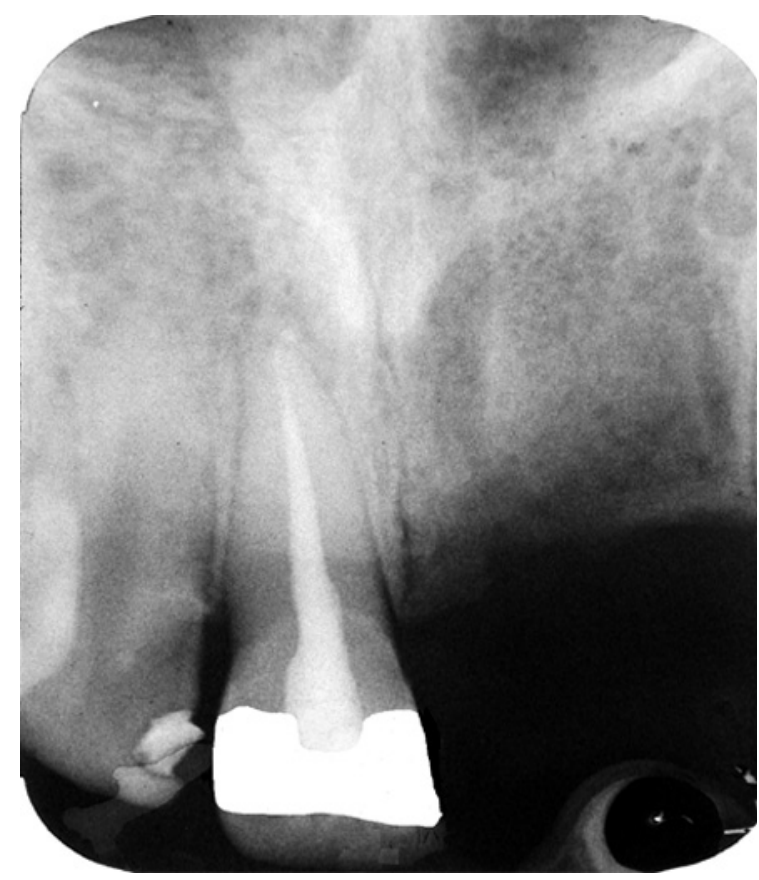

Figure 2 : Radiographie de contrôle à 6 mois ; absence de lésion apicale résiduelle.

Recall X-ray at 6 month; absence of periapical lesion.

\section{Evaluation du résultat}

Les critères diagnostiques du succès clinique et radiographique en endodontie ont permis de constater une évolution vers la guérison de la lésion. Observée à partir du troisième mois de contrôle, elle semble achevée au sixième mois : pas de sensibilité à la percussion et à la palpation, mobilité physiologique, pas de signes d'infection et de tuméfaction, pas de signes subjectifs d'inconfort, espace desmodontal normal, disparition de l'image apicale de raréfaction osseuse, densification des tissus osseux périapicaux, obturation dense confinée à l'espace endodontique [3] (Fig. 2).

\section{COMMENTAIRE}

L'abcès phœnix est une affection d'origine infectieuse qui nécessite un traitement adapté. La maintenance de la chaîne d'asepsie (digue, bains de bouche...) est aussi importante que l'antisepsie du canal radiculaire.

Une antibiothérapie systémique a été prescrite car, lors de la première séance, le drainage de l'abcès n'était pas suffisant et il y existait des signes généraux. L'amoxicilline est un antibiotique adapté à la population bactérienne présente, mais l'on sait que quelques bactéries synthétisent des béta-lactamases : dans ce cas particulier, on a opté pour l'association amoxicilline-acide clavulanique [1].

Le facteur le plus important dans la symptomatologie de l'abcès phœnix est l'élévation de la pression dans les tissus périapicaux : seul le drainage et la normalisation de la pression tissulaire permettent la sédation des douleurs [17]. Le contrôle de l'occlusion est également très important : l'œdème périapical, toujours présent, impose un meulage coronaire sélectif. Le traitement de l'abcès phœnix passe nécessairement par l'élimination la plus complète possible de tous les débris organiques servant de support aux microorganismes présents au sein du système canalaire. II est important de rappeler que la flore endodontique varie dans le temps en fonction de la localisation endocanalaire, des conditions nutritives et de l'oxygénation. Ce sont essentiellement des coques à Gram+ (Peptostreptococcus ssp, médecine

buccale

chirurgie

buccale

VOL. $13, \mathrm{~N}^{\circ} 3$ 2007

page 163 
médecine

buccale

chirurgie

buccale

VOL. $13, \mathrm{~N}^{\circ} 3$ 2007

page 164
Streptococcus ssp, Enterococcus ssp) et des bacilles anaérobies stricts à Gram- (Porphyromonas ssp, Prevotella ssp, Fusobacterium ssp). Ces espèces microbiennes, présentes dans les canaux radiculaires, seront éliminées par des moyens mécaniques et une irrigation à l'hypochlorite de sodium. Les impératifs biologiques imposent de réaliser un nettoyage progressif et d'éviter l'ensemencement bactérien. A cet effet, l'utilisation d'instruments endodontiques à forte conicité, en rotation continue, est préférable à la préparation séquentielle manuelle utilisée, faute de moyen.

La littérature préconise une médication intracanalaire antiseptique. L'hydroxyde de calcium est utilisé pour éliminer de manière prévisible les microorganismes du système canalaire ; la smear layer est enlevée pour faciliter la diffusion de l'hydroxyde de calcium dans les tibuli dentinaires. La plupart des auteurs recommandent l'emploi de l'hydroxyde de calcium à l'état pur car l'adjonction d'autres agents augmente potentiellement le pouvoir antiseptique mais elle provoque une baisse du $\mathrm{pH}$ [15]. Ils préconisent une application de 7 jours alors que l'activité antiseptique optimale est obtenue à partir de 72 heures.

L'activité antiseptique de l'hydroxyde de calcium est due à son $\mathrm{pH}$ alcalin $(\mathrm{pH}=12)$, ce qui lui confère un caractère cytotoxique. Très peu de bactéries résistent à un $\mathrm{pH}$ supérieur à 9,5 [8] et rares sont les bactéries qui survivent à un $\mathrm{pH}$ supérieur à $11^{[4]}$. Les ions $\mathrm{OH}^{-}$de l'hydroxyde de calcium neutralisent l'acidité liée aux ions $\mathrm{H}^{+}$produits par la flore bactérienne. L'hydroxyde de calcium a une double action : il est bactéricide et organolytique. Toutefois, sa faible solubilité limite son action et son efficacité envers Enterococcus faecalis est discutée ${ }^{[7]}$. Outre ses propriétés antiseptiques, d'autres effets biologiques sont à mettre à l'actif de l'hydroxyde de calcium. Son effet anti-inflammatoire est dû au caractère alcalin du matériau et à la diffusion des ions $\mathrm{Ca}^{++}$et
$\mathrm{OH}^{-}$. Cette alcalinité s'oppose à l'acidose liée à l'inflammation. En luttant contre les ostéoclastes, elle inhibe la perte osseuse au bout de 7 jours. L'action antihémorragique est lié à la diffusion d'ion $\mathrm{Ca}^{++}$; l'hémorragie cesse par nécrose de coagulation [13]. L'action anti-exsudative est en rapport avec le caractère anabolisant du $\mathrm{Ca}(\mathrm{OH})_{2}{ }^{[5]}$. Enfin, l'hydroxyde de calcium est un activateur biologique de la minéralisation [6]. A ce sujet, le rôle attribué aux cellules souches est prépondérant dans le cadre de la cicatrisation des lésions périapicales. Ces cellules possèdent certaines caractéristiques que Potten et Loeffler ont décrites en 1990 [12]. Les remaniements tissulaires à partir de la division des cellules souches et la différenciation des cellules filles, après le traitement de l'abcès phœnix, vont permettre réparer ou même de régénérer les tissus d'ancrage atteints par la lésion. Ces événements sont pilotés par une signalisation cellulaire complexe dans laquelle les facteurs de croissance sont très impliqués ${ }^{[9]}$.

\section{CONCLUSION}

L'abcès phœnix, ou abcès récurrent, correspond à la manifestation aiguë survenant sur une infection apicale chronique qui se caractérise par une collection purulente localisée dans le périapex. La démarche thérapeutique repose essentiellement sur le geste opératoire. L'antibiotique n'est qu'un adjuvent et non une alternative au drainage du pus, à la mise en place d'une médication intracanalaire temporaire $\left[\mathrm{Ca}(\mathrm{OH})_{2}\right]$, au nettoyage ou renettoyage du canal associé à une obturation canalaire et coronaire étanche.

Cependant, chez les patients à risque bactérien systémique, c'est en général l'extraction qui est programmée car le traitement endodontique ne peut s'envisager que s'il peut être conduit en une seule séance ${ }^{[17]}$. 


\section{RÉFÉRENCES}

1 - Abott P. Antibiotics and endodontics. Aust Dent J 1990; 35: 50-60.

2 - Adou AJ, Kouame P, Sinan AA. Conserver ou extraire ; l'attitude de l'omnipraticien. Rev Cosa-CMF 2005 ; 12 : 31-4.

3 - Andem. Agence nationale pour le développement et l'évaluation de la Médecine; Service des références dentaires, 1996.

4 - Byström A, Claesson R, Sundqvist G. The antibacterial effect of camphorated paramonochlorophenol, camphorated phenol and calcium hydroxide in the treatment of infected root canals. Endod Dent Traumatol $1985 ; 1: 170-5$.

5 - Chong BS, Pitt TR. The role of intracanal medication in root canal treatment. Int Endod J 1992 ; 25 : 97-106.

6 - Foreman PC, Barnes IE. A review of calcium hydroxide. Int Endod J 1990 ; 23 : 283-97.

7 - Gomez B, Ferraz C, Souza S. In vitro antimicrobial activity of chlorhexidine gel and calcium hydroxide separately and combined in infected bovine root dentin. J Endod $2001 ; 27: 233$.

8 - Heithersay GS. Calcium hydroxide in the treatment of pulpless teeth with associated pathology. J Br Endod Soc $1975 ; 8: 74-93$.
9 - King GN. New regenerative technologies: rationale and potential for periodontal regeneration: growth factors. Dental Update $2001 ; 28$ : 60-5.

10 - Lasfargues JJ. Le diagnostic clinique des parodontites apicales. Réalités cliniques 2001 ; 12 : 149-62.

11 - Machtou P. Guide clinique d'Endodontie. CDP, Paris, 1993.

12 - Potten CS, Loeffler M. Stem cells: attributes, cycles, spirals, pit bulls and uncertainties. Development 1990 ; 110: 1001-20.

13 - Ricci C, Travert V. L'hydroxyde de calcium en endodontie. Rev Fr Endod 1987; 6 : 45-74.

14 - Siqueira JF, Barnet F. Interappointment pain: mechanisms, diagnosis and treatment. Endod Topics 2004; 7: 93-109.

15 - Steven R, Grossman L. Evaluation of the antimicrobial potential of calcium hydroxide as an intracanal medicament, J Endod $2001 ; 9$ : 372-4.

16 - Ten Cate AR. Oral histology. Development, structure and function. Mosby, 1980.

17 - Toumelin-Chemla F. Les urgences endodontiques. Cahier de Formation Médico-Dentaires Continue, Info Dent $1998 ; 1-12$. \begin{tabular}{l}
$\begin{array}{l}\text { médecine } \\
\text { buccale } \\
\text { chirurgie } \\
\text { buccale }\end{array}$ \\
\hline VOL. $13, N^{\circ} 3$ \\
2007
\end{tabular}

page 165 